\title{
Composição fenólica e atividade antioxidante de resíduos agroindustriais
}

\author{
Phenolic composition and antioxidant activity of agroindustrial residues
}

\author{
Priscilla Siqueira Melo ${ }^{\mathrm{I}}$ Keityane Boone Bergamaschi ${ }^{\mathrm{I}}$ Ana Paula Tiveron ${ }^{\mathrm{I}}$ Adna Prado Massarioli ${ }^{\mathrm{I}}$ \\ Tatiane Luiza Cadorin Oldoni ${ }^{\mathrm{I}}$ Mauro Celso Zanus ${ }^{\mathrm{II}}$ Giuliano Elias Pereira' ${ }^{\mathrm{II}}$ \\ Severino Matias de Alencar ${ }^{{ }^{*}}$
}

\section{RESUMO}

Atualmente, são produzidas milhões de toneladas de resíduos provenientes do processamento agroindustrial. Muitos deles são ricos em compostos bioativos sendo potenciais fontes naturais dessas substâncias. Assim, este trabalho teve como objetivo avaliar o teor de compostos fenólicos totais, a atividade antioxidante e a composição fenólica de três resíduos gerados por agroindústrias brasileiras: bagaço de uva Isabel (BI) (Vitis labrusca), bagaço de uva Verdejo (BV) (Vitis vinifera) e bagaço de goiaba (BG) (Psidium guajava). Os resultados do teor de compostos fenólicos totais ( $\mathrm{mg}$ GAE $\mathrm{g}^{-1}$ ) encontrados nos extratos etanólicos e aquosos dos resíduos foram, respectivamente: $\quad B V \quad(20,94 \pm 0,46 ; 8,03 \pm 0,43)>\quad B I$ $(16,57 \pm 0,19 ; 4,41 \pm 0,01)>B G(3,41 \pm 0,09 ; 1,88 \pm 0,06)$. Alta atividade antioxidante, principalmente em $B V$ e $B I$, foi verificada nos ensaios realizados (ABTS •, DPPH $\bullet$ e autooxidação do sistema beta-caroteno/ácido linoléico). Uma forte correlação positiva entre atividade antioxidante e o teor de compostos fenólicos totais foi encontrada. Os compostos fenólicos encontrados, por cromatografia gasosa com espectrometria de massas (CG-EM), foram: ácido gálico, epicatequina, quercetina (BV, BI e BG); ácido isovanílico (BI, $B G)$; ácido p-cumárico (BI); ácido caféico e resveratrol (BV, $B I)$. Esses resultados mostram que os resíduos agroindustriais analisados, particularmente os vinícolas, são ricos em substâncias bioativas e podem ser explorados pela indústria de alimentos e farmacêutica.

Palavras-chave: resíduos agroindustriais, compostos fenólicos, atividade antioxidante, $\mathrm{DPPH} \bullet$, ABTS • , auto-oxidação do sistema betacaroteno/ácido linoléico, CG-EM.

\section{ABSTRACT}

Nowadays, the agro-industrial processing produces millions of tons of wastes. Many of them are rich in bioactive compounds, being a potential natural source of these substances. This study aimed to evaluate the content of total phenolics, antioxidant activity and phenolic composition of residues generated by three Brazilian agribusiness: Isabel grape pomace (PI) (Vitis labrusca), Verdejo grape pomace (PV) (Vitis vinifera) and guava pomace (PG) (Psidium guajava). The results of total phenolics content ( $\mathrm{mg} G A E \mathrm{~g}^{-1}$ ) found in the ethanol and aqueous extracts of residues were: $P V(20.94 \pm 0.46$; $8.03 \pm 0.43)>P I(16.57 \pm 0.19 ; 4.41 \pm 0.01)>P G(3.41 \pm 0.09$; $1.88 \pm 0.06)$. High antioxidant activity of these extracts, particularly $P V$ and $P I$, was found by the methods $D P P H \bullet$, ABTS • and beta-carotene bleaching method. A strong positive correlation between antioxidant activity and content of total phenolic compounds was found. The following phenolic compounds were found by gas chromatography with mass spectrometry (GC-MS): gallic acid, epicatechin, quercetin (PV, $P I$ and $P G)$; isovanilic acid (PI, PG), p-coumaric acid (PI), caffeic acid and resveratrol $(P V, P I)$. The results show that these residues, particularly the wineries, are rich in bioactive substances and should be exploited by the food industry and pharmaceuticals.

Key words: agroindustrial residues, phenolic compounds, antioxidant activity, DPPH •, ABTS • , betacarotene bleaching method, GC-MS.

'Departamento de Agroindústria, Alimentos e Nutrição, Escola Superior de Agricultura "Luiz de Queiroz" (ESALQ), Universidade de São Paulo (USP), Av. Pádua Dias, 11, 13418-900, Piracicaba, SP, Brasil. E-mail: alencar@esalq.usp.br.*Autor para correspondência.

IIEmpresa Brasileira de Pesquisa Agropecuária, EMBRAPA Uva e Vinho, Bento Gonçalves, RS, Brasil.

IIIEmpresa Brasileira de Pesquisa Agropecuária, EMBRAPA Semi Árido, Petrolina, PE, Brasil. 


\section{INTRODUÇÃO}

Em todo o mundo, são geradas milhões de toneladas de resíduos provenientes de atividades agroindustriais (MAKRIS et al., 2007). Alguns deles são aproveitados como ração animal ou dispostos no campo, entretanto, a maior parte ainda é descartada sem tratamento, causando danos ao meio ambiente.

Além disso, o destino dado a esses resíduos, tal como é feito, causa um déficit econômico na cadeia produtiva, uma vez que muitos deles são ricos em compostos bioativos, alguns capazes de combater danos oxidativos causados por radicais livres, como é o caso dos antioxidantes - substâncias de elevado valor comercial. Produzidos como metabólitos secundários de plantas, os antioxidantes possuem larga aplicação nos setores farmacêutico, cosmético e nutricional, além de servirem como aditivos naturais em alimentos, atribuição esta que tem ganhado importância crescente, pois os antioxidantes sintéticos usados pela indústria de alimentos como o BHA (butilhidroxianisol), o BHT (butil-hidroxitolueno) e o TBHQ (terc-butilhidroquinona) despertam preocupação quanto as suas doses de segurança e toxicidade (BALASUNDRAM et al., 2006).

Dentre os diversos resíduos gerados pela agroindústria, destacam-se os vinícolas por serem fontes ricas de compostos fenólicos (RUBILAR et al., 2007) e pela expressiva quantidade resultante do processamento, já que a soma deles, bagaço (cascas e sementes), engaço e a borra do processo fermentativo representam, em média, cerca de $30 \%$ do volume de uvas utilizadas para a produção vinícola (MAKRIS et al., 2007), o que torna este setor uma fonte promissora de substâncias bioativas naturais.

Além dos vinícolas, muitos outros resíduos são gerados pelas atividades agroindustriais. O processamento de goiaba, por exemplo, visando principalmente à produção de sucos, geléias e polpas, implica na geração residual de sementes e parte da polpa. Apesar de a goiaba ser considerada uma boa fonte de antioxidantes e ácido ascórbico (LEONG \& SHUI, 2002), pouco se sabe ainda sobre o valor e/ou potencial de aplicação de seus resíduos (CORREIA et al., 2004).

Baseado no panorama apresentado, este trabalho teve como objetivo avaliar a composição fenólica e o potencial antioxidante de três resíduos agroindustriais (bagaços de uva tinta Isabel e de uva branca Verdejo e bagaço de goiaba), os quais são gerados em quantidades cada vez mais crescentes. Espera-se que os resultados deste estudo sirvam de subsídios à indústria de alimentos para a utilização de antioxidantes naturais em substituição aos sintéticos, resultando em benefícios à saúde humana e, ao mesmo tempo, colaborando para a diminuição do descarte desses resíduos ao meio ambiente.

\section{MATERIAL E MÉTODOS}

O bagaço de uva tinta Isabel (BI) (Vitis labrusca) (Bento Gonçalves, RS), o bagaço de uva branca Verdejo (BV) (Vitis vinifera) (Petrolina, $\mathrm{PE}$ ) e o bagaço de goiaba (Psidium guajava) (BG) (Monte Alto, SP) foram coletados no primeiro semestre de 2009. O transporte dos resíduos foi feito sob refrigeração. Em seguida, eles foram congelados, liofilizados, homogeneizados, pesados e armazenados a $-18^{\circ} \mathrm{C}$.

Os extratos dos resíduos foram obtidos em triplicata, como descrito por BLOOR (2001), com o emprego dos solventes etanol:água $(80: 20 \mathrm{v} / \mathrm{v})$ e água. Inicialmente, pesou-se 1 grama de cada resíduo liofilizado e moído e adicionou-se $10 \mathrm{~mL}$ dos solventes. A extração foi conduzida em ultrassom, à temperatura ambiente, durante 15 minutos. Após isso, o extrato foi centrifugado a $5000 \mathrm{xg}$ durante 15 minutos e o sobrenadante utilizado para as análises.

A análise de compostos fenólicos totais foi feita de acordo com o método de Folin-Ciocalteau (SINGLETON et al.,1999), utilizando ácido gálico como padrão. A atividade antioxidante pelo método de redução do radical ABTS [2,2' -azinobis(3-etilbenzotiazolina-6ácido sulfônico)] foi feita conforme metodologia descrita pela EMBRAPA (2007). A atividade sequestradora do radical DPPH foi realizada de acordo com a metodologia descrita por MENSOR et al. (2001). A medida da atividade antioxidante pela oxidação acoplada do beta-caroteno/ ácido linoléico foi realizada de acordo com o método de EMMONS et al. (1999).

Previamente à análise por Cromatografia gasosa acoplada com espectrometria de massas (CGEM), os extratos dos resíduos foram purificados pela técnica de SPE (Solid Phase Extraction), para eliminação de interferentes, principalmente açúcares, usando cartuchos SPE-LC18 (Supelco, 2 gramas). Às frações obtidas na purificação foram adicionados $100 \mu \mathrm{L}$ de N-metil-N-(trimetilsilil)-trifluoroacetamida (MSTFA) para derivatização. As amostras silanizadas foram analisadas em um cromatógrafo gasoso (Shimadzu GC 2010) acoplado a espectrômetro de massas (Shimadzu QP 2010 Plus) e equipado com uma coluna capilar (RTX5MS 30mx0,25mmx0,25 $\mu \mathrm{m}$ ). A programação de temperatura iniciou em $80^{\circ} \mathrm{C}$ (1 minuto), a uma taxa de aquecimento de $20^{\circ} \mathrm{C}$ minuto $^{-1}$ alcançou $250^{\circ} \mathrm{C}(1$ minuto), passou a $300^{\circ} \mathrm{C}$ ( 5 minutos) a uma taxa de $6^{\circ} \mathrm{C}$ minuto $^{-1}$, a $310^{\circ} \mathrm{C}(5$ minutos $)$ a uma taxa de $15^{\circ} \mathrm{C} \mathrm{minuto}^{-1}$ e a $320^{\circ} \mathrm{C}$ (10 minutos) a uma taxa de $20^{\circ} \mathrm{C}_{\text {minuto }}{ }^{-1}$, 
totalizando 40 minutos de análise. Hélio foi utilizado como gás de arraste. A temperatura do injetor foi de $280^{\circ} \mathrm{C}$ e o volume de injeção de $0,5 \mu \mathrm{L}$ no modo splitless. A interface foi mantida a $280^{\circ} \mathrm{C}$ e o detector operou no modo scanning ( $\mathrm{m} / \mathrm{z}$ 40-800) (PROESTOS et al., 2006, com modificações). Os compostos fenólicos foram identificados por comparação com dados obtidos do CG-EM (tempo de retenção e fragmentação iônica) de padrões autênticos Extrasynthese Co. (resveratrol, ácido siríngico, miricetina, kaempferol, luteolina, liquiritigenina, isoliquiritigenina, quercetina, miricetina, ácido $p$-cumárico, ácido ferúlico, catequina, epicatequina) e com a biblioteca Wiley 8 .

Todas as análises foram conduzidas em triplicata e os resultados apresentados como média seguidos do desvio padrão. A análise estatística foi feita pela aplicação do teste de Tukey $(\mathrm{P}<0,05)$, usando o software SAS (2002).

\section{RESULTADOS E DISCUSSÃO}

Os extratos etanólicos apresentaram os maiores teores de compostos fenólicos, demonstrando assim que esse solvente foi mais eficiente na extração dessa classe de substâncias químicas (Tabela 1). O bagaço de uva Verdejo (BV) apresentou o maior conteúdo de compostos fenólicos totais dentre as amostras analisadas, superando, inclusive, o bagaço tinto de uva Isabel (BI) que, por sua vez, apresentou maior teor do que o bagaço de goiaba (BG). MAKRIS e colaboradores (2007) analisaram a composição fenólica de bagaços de uva branca, tinta e engaço e encontraram teores, equivalentes em ácido gálico, de $48,26 \mathrm{mg} \mathrm{g}^{-1}$ para bagaço de uva branca e $54,02 \mathrm{mg} \mathrm{g}^{-1}$ para bagaço de uva tinta, valores esses superiores aos encontrados neste trabalho. Diferenças entre variedades, região geográfica, safra, condições climáticas, peculiaridades

Tabela 1 - Teor de compostos fenólicos totais (mg GAE $\mathrm{g}^{-1}$ ) dos extratos dos resíduos agroindustriais.

\begin{tabular}{lcc}
\hline Resíduos agroindustriais & \multicolumn{2}{c}{ Compostos fenólicos ${ }^{(\mathrm{a}, \mathrm{b}, \mathrm{c})}$} \\
\hline & Etanol $80 \%$ & Água \\
BV & $20,94 \pm 0,46 \mathrm{a}$ & $8,03 \pm 0,43 \mathrm{c}$ \\
BI & $16,57 \pm 0,19 \mathrm{~b}$ & $4,41 \pm 0,01 \mathrm{~d}$ \\
BG & $3,41 \pm 0,09 \mathrm{e}$ & $1,88 \pm 0,06 \mathrm{f}$ \\
\hline
\end{tabular}

Legenda: $\mathrm{BV}=$ Bagaço Verdejo; $\mathrm{BI}=$ Bagaço Isabel; $\mathrm{BG}=$ Bagaço Goiaba.

${ }^{a}$ Média \pm desvio padrão da triplicata da extração.

${ }^{\mathrm{b}}$ Médias seguidas de mesma letra não diferem estatisticamente entre si pelo teste de Tukey $(\mathrm{P}<0,05)$.

${ }^{\mathrm{c}}$ Equivalentes em ácido gálico. de processamento e métodos de extração podem interferir nos resultados do teor de compostos fenólicos, bem como, na expressão da atividade antioxidante de materiais vegetais (ROCKENBACH et al., 2008; RUBERTO et al., 2007).

Os extratos etanólicos do BV e BI que tiveram os maiores teores de compostos fenólicos totais também apresentaram as maiores atividades antioxidantes pelo método ABTS • (Figura 1A), com exceção do extrato aquoso BG, que, apesar de possuir um baixo teor de compostos fenólicos (Tabela 1), não apresentou diferença significativa da atividade antioxidante em relação à apresentada pelo extrato etanólico BG. Estudo feito por ROCKENBACH et al. (2008) mostrou que extratos etanólicos (etanol:água; 70:30; v/v) de bagaços de uva das variedades Ancelota e Tannat apresentaram, pelo método ABTS $\bullet$, atividade antioxidante em equivalentes de trolox de $389,9 \pm 3,8$ e $398,1 \pm 2,8 \mu \mathrm{mol}$ trolox $\mathrm{g}^{-1}$, respectivamente. Alto potencial antioxidante pelo método ABTS • para bagaços de uva também foi encontrado por RUBERTO et al. (2007).

No ensaio da atividade sequestrante do radical livre DPPH $\bullet$, todos os extratos, tanto aquosos quanto etanólicos, apresentaram alta atividade antioxidante (Figura 1B). Elevados potenciais de redução do DPPH • também foram observados por outros autores(RUBERTO etal., 2007; MAKRIS etal., 2007; LAFKA et al., 2007) para extratos de bagaços de uva.

Com relação à análise da atividade antioxidante pela auto-oxidação do sistema betacaroteno/ácido linoléico, não foi verificada diferença significativa entre os extratos etanólico e aquoso do resíduo BV e extrato etanólico de $\mathrm{BI}$ (Figura 1C). O menor percentual antioxidante foi o apresentado pelo extrato etanólico de BG.

A variação da expressão da atividade antioxidante por esses diferentes métodos sugere que os compostos fenólicos desses resíduos exercem atividades antioxidantes por mecanismos de ação distintos, dependendo da polaridade do meio reacional. Isso significa dizer que os extratos etanólicos dos resíduos $\mathrm{BV}$ e $\mathrm{BI}$ possuem alta capacidade de sequestrar os radicais livres ABTS e DPPH, enquanto que os extratos aquosos dos resíduos $\mathrm{BV}$ e $\mathrm{BG}$ possuem potencial de proteção de um substrato lipídico da oxidação igual ou superior aos seus extratos etanólicos, respectivamente.

A auto-oxidação do beta-caroteno/ácido linoléico é um ensaio que difere dos outros dois métodos de avaliação da atividade antioxidante $(\mathrm{ABTS} \bullet$ e DPPH•), pois sua matriz de reação é uma emulsão. O comportamento de muitos antioxidantes pode mudar ante o meio em que se encontram. Em 


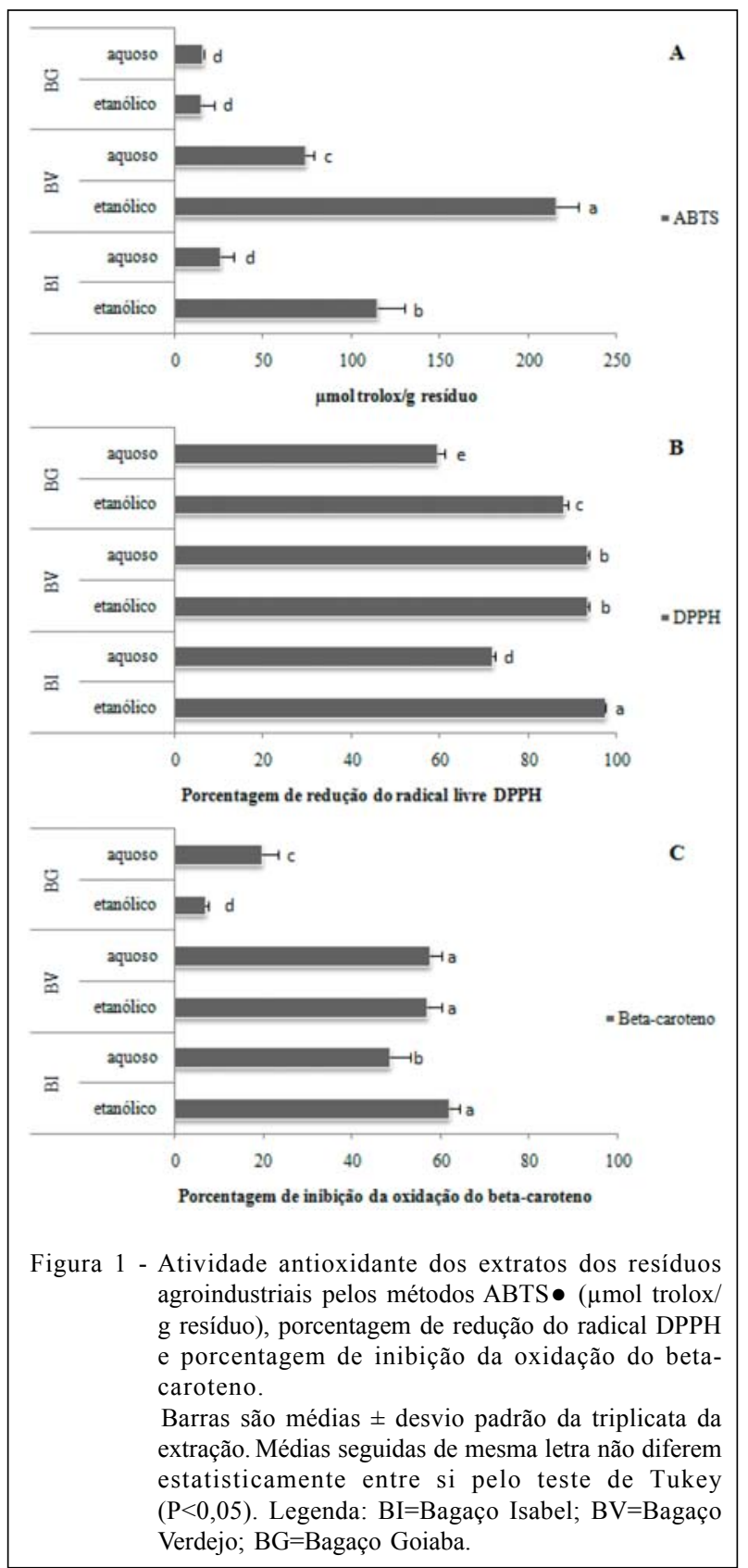

emulsões, é relatada maior eficácia protetora de antioxidantes lipofílicos, já que se direcionam na interface óleo-água (KIOKIAS et al., 2008). Entretanto, considerando o perfil polar de compostos fenólicos e a correlação moderada verificada com a atividade antioxidante pelo método da auto-oxidação do betacaroteno $(\mathrm{r}=0,713)$ (Tabela 2), outros compostos químicos não identificados neste trabalho podem ter contribuído para a atividade antioxidante dos extratos aquosos por esse método.
Tabela 2 - Coeficientes de correlação (r) entre atividade antioxidante e alguns compostos fenólicos dos extratos dos resíduos agroindustriais.

\begin{tabular}{lc}
\hline \multicolumn{1}{c}{ Correlação } & $\mathrm{R}$ \\
\hline Compostos fenólicos x ABTS & 0,967 \\
Compostos fenólicos x DPPH & 0,705 \\
Compostos fenólicos x beta-caroteno & 0,713 \\
Compostos fenólicos identificados nos extratos etanólicos \\
Ácido gálico x ABTS & 0,530 \\
Ácido gálico x DPPH & 0,017 \\
Ácido gálico x beta-caroteno & 0,040 \\
Epicatequina x ABTS & 0,342 \\
Epicatequina x DPPH & 1,000 \\
Epicatequina x beta-caroteno & 0,894 \\
Quercetina x ABTS & 0,672 \\
Quercetina x DPPH & 0,889 \\
Quercetina x beta-caroteno & 1,000 \\
Compostos fenólicos identificados nos extratos aquosos \\
Ácido gálico x ABTS & 0,646 \\
Ácido gálico x DPPH & 0,828 \\
Ácido gálico x beta-caroteno & 0,999 \\
Epicatequina x ABTS & 0,313 \\
Epicatequina x DPPH & 0,518 \\
Epicatequina x beta-caroteno & 0,898 \\
Quercetina x ABTS & 0,282 \\
Quercetina x DPPH & 0,117 \\
Quercetina x beta-caroteno & 0,008 \\
\hline & \\
\hline
\end{tabular}

A atividade antioxidante dos extratos dos resíduos agroindustriais por esses diferentes métodos de avaliação apresentou uma correlação alta e positiva com o conteúdo de compostos fenólicos totais (Tabela 2). A maior correlação verificada foi para o teste do ABTS $(r=0,9673)$. Isso sugere que os compostos fenólicos presentes nesses resíduos possuem alta capacidade de sequestro de radicais livres e, por isso, são fontes importantes de agentes antioxidantes primários, de grande importância para a indústria de alimentos.

A composição fenólica dos extratos etanólicos e aquosos dos resíduos identificados pela técnica de CG-EM está apresentada na tabela 3. Notase que, em todos os bagaços derivados de uva, tanto branco quanto tinto, houve a predominância do flavonóide epicatequina, sugerindo que esse composto participa de forma expressiva na atividade antioxidante destas amostras. De fato, nos extratos etanólicos, a epicatequina mostrou forte correlação com os ensaios de $\mathrm{DPPH} \bullet$ e da auto-oxidação do beta-caroteno e, nos extratos aquosos, com a auto-oxidação do betacaroteno (Tabela 2). Destaca-se ainda a presença de ácido gálico, caféico, ferúlico, vanílico, $p$-cumárico, 
Tabela 3 - Tempos de retenção (em minutos), porcentagem de área de cada componente e íons importantes do espectro de massa dos compostos fenólicos silanizados presentes nos extratos etanólico e aquoso dos resíduos agroindustriais, por CG-EM.

\begin{tabular}{|c|c|c|c|c|c|}
\hline \multirow{3}{*}{ Compostos } & \multicolumn{5}{|c|}{ Porcentagem de área (\%) } \\
\hline & $\mathrm{tR} \min$ & \multicolumn{3}{|c|}{ Extratos etanólicos ${ }^{\mathrm{E}} /$ aquosos $^{\mathrm{A}}$} & \multirow[t]{2}{*}{ Íon ( $m / z$, abundância entre parênteses) } \\
\hline & & BI & BV & BG & \\
\hline Ácido 2-hidroxibenzóico & 7,43 & $\begin{array}{ll}- \\
0,05^{\mathrm{A}}\end{array}$ & $\begin{array}{l}- \\
-\end{array}$ & - & $\begin{array}{l}73(100), 267(75), 232(26), 268(20), 45 \text { (18); } \\
282\left(\mathrm{M}^{+}\right)\end{array}$ \\
\hline Ácido isovanílico & 8,88 & $\begin{array}{l}0,32^{\mathrm{E}} \\
0,47^{\mathrm{A}}\end{array}$ & - & $\begin{array}{l}0,35^{\mathrm{E}} \\
-\end{array}$ & $\begin{array}{l}73(100), 217(57), 297 \text { (49), } 267 \text { (46), } 253 \text { (32); } \\
312\left(\mathrm{M}^{+}\right)\end{array}$ \\
\hline Ácido vanílico & 8,88 & $\begin{array}{l}- \\
-\end{array}$ & - & - & $\begin{array}{l}73(100), 297(91), 267(60), 312(46), 223(44) \text {; } \\
312\left(\mathrm{M}^{+}\right)\end{array}$ \\
\hline $\begin{array}{l}\text { Ácido } \quad 2,4 \\
\text { dihidroxibenzóico }\end{array}$ & 8,93 & - & - & - & $\begin{array}{l}355(100), 73(92), 356(32), 357 \text { (17), } 45 \text { (14); } \\
371\left(\mathrm{M}^{+}\right)\end{array}$ \\
\hline Ácido m-cumárico & 9,8 & - & - & $\begin{array}{l}1,02^{\mathrm{E}} \\
-\end{array}$ & $\begin{array}{l}73(100), 324(51), 293(36), 204(26), 294(25) \text {; } \\
308\left(\mathrm{M}^{+}\right)\end{array}$ \\
\hline Ácido-p-cumárico & 9,8 & $\begin{array}{l}0,74^{\mathrm{E}} \\
1,31^{\mathrm{A}}\end{array}$ & - & $\begin{array}{l}- \\
-\end{array}$ & $\begin{array}{l}73(100), 293(59), 219 \text { (54), } 308 \text { (45), } 249 \text { (38); } \\
308\left(\mathrm{M}^{+}\right)\end{array}$ \\
\hline Ácido gálico & 9,89 & $\begin{array}{l}2,07^{\mathrm{E}} \\
1,36^{\mathrm{A}}\end{array}$ & $\begin{array}{l}8,59^{\mathrm{E}} \\
1,83^{\mathrm{A}}\end{array}$ & $\begin{array}{l}3,65^{\mathrm{E}} \\
-\end{array}$ & $\begin{array}{l}281(100), 73(97), 458(64), 443(28), 459 \text { (25); } \\
458\left(\mathrm{M}^{+}\right)\end{array}$ \\
\hline Ácido ferúlico & 10,7 & - & $\begin{array}{l}0,18^{\mathrm{E}} \\
-\end{array}$ & - & $\begin{array}{l}338(100), 73(78), 308(54), 323(53), 249 \text { (39); } \\
338\left(\mathrm{M}^{+}\right)\end{array}$ \\
\hline Ácido caféico & 10,95 & $\begin{array}{l}0,82^{\mathrm{E}} \\
1,77^{\mathrm{A}}\end{array}$ & $\begin{array}{l}0,20^{\mathrm{E}} \\
-\end{array}$ & - & $\begin{array}{l}219(100), 73(82), 396(76), 397(27), 381 \text { (21); } \\
396\left(\mathrm{M}^{+}\right)\end{array}$ \\
\hline Resveratrol & 15,74 & $0,94^{\mathrm{E}}$ & $\begin{array}{l}0,40^{\mathrm{E}} \\
-\end{array}$ & - & $\begin{array}{l}444(100), 73(84), 445(36), 446(18), 443(13) \text {; } \\
444\left(\mathrm{M}^{+}\right)\end{array}$ \\
\hline Epicatequina (pico 1) & 17,2 & $\begin{array}{l}19,17^{\mathrm{E}} \\
25,50^{\mathrm{A}}\end{array}$ & $\begin{array}{l}3,43^{\mathrm{E}} \\
7,54^{\mathrm{A}}\end{array}$ & - & $\begin{array}{l}368(100) ; 73(93), 307(43), 355(39), 369 \text { (31); } \\
650\left(\mathrm{M}^{+}\right)\end{array}$ \\
\hline Epicatequina (pico 2) & 17,43 & $\begin{array}{l}54,26^{\mathrm{E}} \\
42,33^{\mathrm{A}}\end{array}$ & $\begin{array}{l}40,27^{\mathrm{E}} \\
53,78^{\mathrm{A}}\end{array}$ & $\begin{array}{l}0,67^{\mathrm{E}} \\
1,96^{\mathrm{A}}\end{array}$ & $\begin{array}{l}368(100) ; 73(64), 355(36), 369(29), 370(13) ; \\
650\left(\mathrm{M}^{+}\right)\end{array}$ \\
\hline Quercetina & 20,6 & $\begin{array}{l}0,37^{\mathrm{E}} \\
0,38^{\mathrm{A}}\end{array}$ & $\begin{array}{l}0,75^{\mathrm{E}} \\
1,30^{\mathrm{A}}\end{array}$ & $\begin{array}{l}4,56^{\mathrm{E}} \\
1,11^{\mathrm{A}}\end{array}$ & $\begin{array}{l}647(100), 648(57), 73(68), 649(33), 650(13) ; \\
662\left(\mathrm{M}^{+}\right)\end{array}$ \\
\hline
\end{tabular}

resveratrol, quercetina que são reconhecidos por possuírem atividade antioxidante, seja de forma independente ou por meio de sinergismo. Muitos desses compostos também foram identificados pela técnica de cromatografia líquida de alta eficiência em estudos feitos por LAFKA et al. (2007) para bagaço de uva Agiorgitiko, RUBILAR et al. (2007) para bagaço de Cabernet Sauvignon e por THIMOTHE et al. (2007) para bagaços de Pinot Noir, Cabernet Franc, Baco Noir e Noiret.

\section{CONCLUSÃO}

Os resultados mostram que os resíduos agroindustriais analisados, principalmente os vinícolas, são ricos em compostos bioativos, dentre os quais compostos fenólicos, como pôde ser confirmado pela análise em CG-EM. Esses resíduos possuem atividade antioxidante e, por conseguinte, são potenciais fontes naturais de substâncias bioativas para aplicação na indústria de alimentos.

\section{AGRADECIMENTOS}

À Fundação de Amparo à Pesquisa do Estado de São Paulo - FAPESP, pelo auxílio financeiro (Processo n.2008/ 55492-7).

\section{REFERÊNCIAS}

BALASUNDRAM, N. et al. Phenolic compounds in plants and agri-industrial by-products: antioxidant activity, occurrence and potencial uses. Food Chemistry, Barking, v.99, p.191-203, 2006. Disponível em: <http://www.sciencedirect.com/ science?_ob=ArticleURL\&_udi=B6T6R-4H877SK$1 \&$ user $=10 \&$ coverDate $=12 \% 2$ F $31 \% 2$ F $2006 \&$ 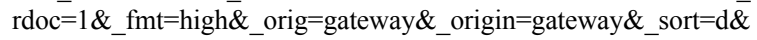 docanchor $=\&$ view $=$ c \&_searchStrId $=1708498179 \&$ rerunOrigi $\overline{\mathrm{n}}=$ google\&_acct $=\mathrm{C} 000050221$ \&_version $=1 \&$ \&urlVersion $=0$ \&_userid $=10 \& \mathrm{md}$ $5=\mathrm{be} 0 \mathrm{~b} 7 \mathrm{f} 4853 \mathrm{~d} 3 \mathrm{a} 6 \mathrm{~d} 05 \mathrm{f} 37 \mathrm{a} 31121333 \mathrm{a} 95 \&$ searchtype $=\mathrm{a} ? ?>$. Acesso em: 08 jul. 2010. doi: 10.1016/j.foodchem.2005.07.042. 
BLOOR, S.J. Overview of methods for analysis and identification of flavonoids. Methods in Enzymology, New York, v.335, p 3-14, 2001. Disponível em: <http://

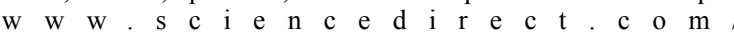
science? ob=ArticleURL\& udi=B 7CV2-4B263HS$2 \&$ \&user $=10 \&$ \&coverDate $=1 \overline{2} \% 2$ F $31 \% 2$ F $2001 \&$ rdoc $=1 \&$ _fmt $=$ high \&_orig $=$ gateway\&_origin $=$ gateway\&_sort $=\mathrm{d} \&$ doc anchor $=\&$ vi e w $=\mathrm{c} \&$ s e a r c h S tr I d $=1708$ $\overline{5} 17281 \&$ \& e r un Orig i n $=$ g o o g le \&_a c c t $=\mathrm{C} 00005022 \overline{1} \&$ version $=1 \&$ urlVersion $=0 \&$ userid $=1$ $0 \& \mathrm{md} 5=59865061 \overline{\mathrm{e}} 6 \mathrm{e} 8 \mathrm{e} 4403 \mathrm{~b} 797 \mathrm{f} 2888 \mathrm{~d} 971 \mathrm{~b} 9 \&$ searchtype $=\mathrm{a}>$. Acesso em: 25 maio, 2010. doi: 10.1016/S00766879(01)35227-8.

CORREIA, R.T.P. et al. Phenolic antioxidant enrichment of soy flour-supplemented guava waste by Rhizopus oligosporus mediated solid-state bioprocessing. Journal of Biochemistry, Tokyo, v.28, p 404-418, 2004. Disponível em: <http:// onlinelibrary.wiley.com/doi/10.1111/j. 1745 4514.2004.05703.x/abstract>. Acesso em: 14 ago. 2009. doi: 10.1111/j.1745-4514.2004.05703.x.

Empresa Brasileira de Pesquisa Agropecuária - EMBRAPA. Metodologia científica: determinação da atividade antioxidante total em frutas pela captura do radical livre ABTS. Fortaleza: Embrapa Agroindústria Tropical, 2007. 4p. (Comunicado técnico, 128).

EMMONS, C.L. et al. Antioxidant capacity of oat (Avena sativa L.) extracts. 2 . In vitro antioxidant activity and content of phenolic and tocol antioxidants. Journal of Agricultural and Food Chemistry, Easton, v.47, p.4894-4898, 1999. Disponível em: <http://pubs.acs.org/doi/abs/10.1021/ jf990530i>. Acesso em: 18 out. 2009. doi: 10.1021/jf990530i.

KIOKIAS, S. et al. In vitro activity of vitamins, flavonoids, and natural phenolic antioxidants against the oxidative deterioration of oil-based systems. Critical Reviews In Food Science And Nutrition, Boca Raton, v.48, n.1, p.78-93, 2008. Disponível em: <http://www.informaworld.com/smpp/ content $\sim \mathrm{db}=\mathrm{all} \sim$ content $=\mathrm{a} 790617639>$. Acesso em: $05 \mathrm{jan}$. 2009. doi: 10.1080/10408390601079975.

LAFKA, T.I. et al. On the extraction and antioxidant activity of phenolic compounds from winery wastes. Food Chemistry, Barking, v.104, p.1206-1214, 2007. Disponível em: <http:// infolib.hua.edu.vn/Fulltext/ChuyenDe2009/CD213/70.pdf $>$. Acesso em: 07 dez. 2008. doi:10.1016/j.foodchem.2007.01.068.

LEONG, L.P.; SHUI, G. An investigation of antioxidant capacity of fruits in Singapore markets. Food Chemistry, Barking, v.76, p.69-75, 2002. Disponível em: <http://www.sciencedirect.com/ science?_ob=ArticleURL\&_udi=B6T6R-44KW0MJB \&_us e r $=10 \&$ \& o ve r D a t e = $01 \% 2$ F $31 \% 2$ F $2002 \&$ rdoc $=1$ \&_fmt $=$ high \&_orig $=$ gatew a

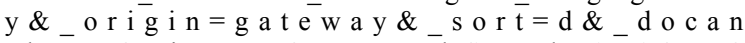
$\mathrm{chor}=\& \mathrm{vi}$ e w $=\mathrm{c} \& \quad \mathrm{~s}$ e a r c h S t r I d $=1 \overline{7} 08573$ $885 \&$ \&erunOrigin $=$ google\&_acct $=\mathrm{C} 000050221 \&$ version $=1 \&$ \& ur 1 Version $=\overline{0} \&$ _us erid $=10 \&$ m d 5 $=0476 \mathrm{c} 1 \mathrm{fc} 65 \mathrm{fd} 51 \mathrm{f} 4 \mathrm{f} 0 \mathrm{f} 25 \mathrm{ecb} 482 \mathrm{~d} 45 \mathrm{da} \&$ searchtype $=\mathrm{a}>$. Acesso em: 15 abr. 2009. doi: 10.1016/S0308-8146(01)00251-5.

MAKRIS, D.P. et al. Polyphenolic content and in vitro antioxidant characteristics of wine industry and other agri-food solid waste extracts. Journal of Food Composition and Analysis, San Diego, v.20, p.125-132, 2007. Disponível em: <http:// www.sciencedirect.com/science?_ob=ArticleURL\&_udi=B6WJH4 KSSW $96-1 \&$ user $=10 \&$ coverDate $=03 \% 2$ F $31 \% 2$ F 20 $07 \&$ rdoc $=1 \&$ fmt $=$ high $\&$ _orig $=$ gateway $\&$ _origin $=$ gatew ay $\&$ sort $=\mathrm{d} \&$ docanchor $=\&$ view $=\mathrm{c} \&$ searchStrId $=1$
$708573138 \&$ \&erunOrigin $=$ google \&_acct $=\mathrm{C} 000050221$ $\&$ version $=1 \&$ urlVersion $=0 \&$ userid $=10 \& \mathrm{md} 5=\mathrm{d}$ 9544c5900e6d2a3a5331f2e06423951\&searchtype $=\mathrm{a}>$. Acesso em: 18 maio, 2009. doi: 10.1016/j.jfca.2006.04.010.

MENSOR, L.L. et al. Screening of brazilian plant extracts for antioxidant activity by the use of DPPH free radical method. Phytotherapy Research, London, v.15, p.127-130, 2001. Disponível em: <http://onlinelibrary.wiley.com/doi/10.1002/ ptr.687/abstract $>$. Acesso em: 23 set. 2009. doi: 10.1002/ ptr. 687 .

PROESTOS, C. et al. Analysis of ?avonoids and phenolic acids in Greek aromatic plants: Investigation of their antioxidant capacity and antimicrobial activity. Food Chemistry, Barking, v.95, p.664-671, 2006. Disponível em: <http:// www.sciencedirect.com/science?_ob=ArticleURL\&_udi=B6T6R$4 \mathrm{FSCMGB}-\mathrm{F} \&$ user $=10 \&$ coverDate $=04 \% 2 \mathrm{~F} 30 \% 2 \mathrm{~F} 2$ $006 \&$ rdoc $=1 \&$ fmt $=$ high \&_orig $=$ gateway $\&$ origin $=$ gateway 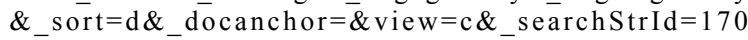 $8 \overline{5} 76787 \&$ rerunOrigin $=$ google \& a c c t $=\mathrm{C} 00$ $0050221 \&$ \&ersion $=1 \&$ \& urlVersion $=0 \&$ _userid $=10 \&$ $\mathrm{md} 5=\mathrm{f} 4 \mathrm{aa} 8599 \mathrm{a} 079 \mathrm{baa} 01 \mathrm{aea} 95 \mathrm{abdb} 1 \mathrm{e} 839 \mathrm{a} \&$ searchtype $=\mathrm{a}>$. Acesso em: 24 jan. 2010. doi:10.1016/j.foodchem.2005.01.049.

ROCKENBACH, I.I. et al. Influência do solvente no conteúdo total de polifenóis, antocianinas e atividade antioxidante de extratos de bagaço de uva (Vitis vinifera) variedades Tannat e Ancelota. Ciência e Tecnologia de Alimentos, Campinas, v.28, p.238-244, 2008. Disponível em: <http:/www.scielo.br/scielo.php?pid=S010120612008000500036\&script=sci_arttext $>$. Acesso em: 18 mar. 2010. doi: 10.1590/S0101-20612008000500036.

RUBERTO, G. et al. Polyphenol constituents and antioxidant activity of grape pomace extracts from five Sicilian red grape cultivars. Food Chemistry, Barking, v.100, p.203-210, 2007. Disponível em: <http://www.sciencedirect.com/ science?_ob=ArticleURL\&_udi=B6T6R-4HK03GR$1 \&$ user $=10 \&$ coverDate $=12 \% 2$ F $31 \% 2$ F $2007 \&$ rdoc 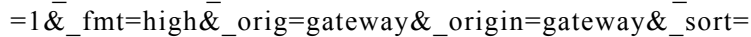 d\&_docanchor $=\&$ view $=$ c \&_searchStrId $=1708581$ $247 \&$ rerunOrigin $=$ google \& acct $=\mathrm{C} 000050221 \&$ ver sion $=1 \&$ \&urlVersion $=0 \&$ userid $=10 \& \mathrm{md} 5=5$ ae $053 \mathrm{e}$ f6916b9f00839b8dbddd6a86d $\&$ searchtype $=a>$. Acesso em: 28 ago. 2009. doi: 10.1016/j.foodchem.2005.09.041.

RUBILAR, M. et al. Separation and HPLC-MS identification of phenolic antioxidants from agricultural residues: almond hulls and grape pomace. Journal of Agricultural and Food Chemistry, Easton, v.55, p.10101-10109, 2007. Disponível em: <http://pubs.acs.org/doi/abs/10.1021/jf0721996>. Acesso em: 25 abr. 2009 . doi: 10.1021/jf0721996.

SAS. SAS INSTITUTE. Statistical Analysis System. User's Guide. Version 9.0. Cary, 2002. 86p.

SINGLETON, V.L. et al. Analysis of total phenols and other oxidation substrates and antioxidants by means of FolinCiocalteau reagent. Methods of Enzymology, New York, v.299, p.152-178, 1999.

THIMOTHE, J. et al. Chemical characterization of red wine grape (Vitis vinifera and Vitis interspecific hybrids) and pomace phenolic extracts and their biological activity against Streptococcus mutans. Journal of Agricultural and Food Chemistry, Easton, v.55, p.10200-10207, 2007. Disponível em: <http://pubs.acs.org/doi/abs/10.1021/jf0722405>. Acesso em: 11 jun. 2010 . doi: $10.1021 /$ jf0722405. 